\title{
Experiments and FE Analyses on Airborne Sound Properties of Composite Structural Insulated Panels
}

\author{
Adam WAWRZYNOWICZ, Marek KRZACZEK, Jacek TEJCHMAN \\ Faculty of Civil and Environmental Engineering, Gdańsk University of Technology \\ Narutowicza 11/12, 80-233 Gdańsk, Poland; \\ e-mail: wawrzynowicz@yahoo.com,\{mkrzacze, tejchmk\}@pg.gda.pl
}

(received May 12, 2013; accepted April 24, 2014)

\begin{abstract}
Airborne acoustic properties of composite structural insulated panels CSIPs composed of fibremagnesium-cement facesheets and expanded polystyrene core were studied. The sound reduction ratings were measured experimentally in an acoustic test laboratory composed of two reverberation chambers. The numerical finite element (FEM) model of an acoustic laboratory available in ABAQUS was used and verified with experimental results. Steady-state and transient FE analyses were performed. The 2D and 3D modelling FE results were compared. Different panel core modifications were numerically tested in order to improve the airborne sound insulation of CSIPs.
\end{abstract}

Keywords: acoustic properties, composite structural insulated panel, sound insulation, finite element method, steady-state analysis, transient analysis.

\section{Introduction}

The structural design in housing can be improved through the development and application of composite elements that capitalize on multifunctional components (PETERs, 1998). Structural insulated panels (SIPs) developed nearly 75 years ago are high-performance composite building panels used in floors, walls, and roofs for residential and light commercial buildings. These panels are fabricated in a factory and shipped to a construction site, where they can be quickly assembled to form a tight, energy-efficient building envelope. SIPs are simple composite sandwich panels formed as three layered structures by bonding a thin layer (facing) to each side of a thick layer (core). The facesheets carry the bending stresses while core resists the shear loads and stabilizes the faces against buckling or wrinkling. The core also increases the structure stiffness by holding the facesheets apart. In general, core materials have lower mechanical properties compared to those of facesheets. The final product has enhanced and more desirable properties than its constituents and possess a high stiffness-to-weight ratio.

In the paper we investigated the last generation of SIPs applied lately in the building industry, called composite structural insulated panels CSIPs (Smakosz et al., 2012; Smakosz, Tejchman, 2014).
They were developed 5 years ago in USA and introduced in 2010 into the Polish building market by the polish building company LS TECH Homes. The panels consist of the glass fibre-magnesium-cement boards (facesheets) with the thickness of $11 \mathrm{~mm}$ and expanded polystyrene EPS core (thickness $150 \mathrm{~mm}$ ) connected by thin adhesive layers. Their dimensions are $0.172 \times 1.0 \times 2.5 \mathrm{~m}^{3}$ and their overall weight is $70 \mathrm{kG}$ As compared to classical SIPs, they are significantly stronger and fire and biological corrosion resistant. Unfortunately, the high stiffness-to-mass ratio causes a low level of the sound insulation due to the appearance of resonant frequencies in the EPS core.

A negative effect of noise on the human health and comfort causes a growing interest in the sound insulation of building baffles. This fact enforces designers to ensure not only the suitable both structural strength and heat-thermal conditions but also the acoustic insulation level. All building standards require to be defined acoustic properties of the building and its components. The most realistic data on the airborne acoustic insulation are obtained by means of field and laboratory acoustic tests. As compared to field measurements, the experimental results from laboratory tests (ISO 140-1, 1997; ISO 140-3, 1995) do not provide data about flanking transmission of the airborne sound through building baffles (ISO 140-4, 1998). 
There exist numerous studies concerning the acoustic behaviour of composite building panels. The first research results on the optimum design of composite panels (LANG, Dym, 1975; MAKris et al., 1986) has shown that the use of more massive and less stiff panels is advantageous for the sound insulation improvement of the sandwich panels over a greater frequency range. Moreover, by increasing the stiffness of the core, the symmetric coincidence effect may be eliminated (while the asymmetric coincidence of the panel still appears). WANG et al. (2009) has shown that the asymmetric coincident frequency of sandwich panels is mainly governed by its flexural stiffness while the symmetric coincident frequency is influenced by the core compressive stiffness. Moreover, the high stiffness-mass ratio is responsible for improving the sound radiation and the lower sound insulation property. The theoretical study by SHENGCHun et al. (2010) indicated that as the core shear stiffness increased, the coincidence critical frequency moved to the lower frequency range and the sound insulation was improved in a medium and high frequency range. A number of tests on samples with different facesheets were carried out by WeNChao and Chung-Fai (1998) to study the effect of the stiffness and damping on the sound insulation of honeycomb sandwich panels. The test results showed that attaching the honeycomb core directly to the interior side of facesheets improved the sound insulation in a low frequency range. Another kind of a honeycomb sandwich panel was presented by NAIFY et al. (2011). The impedance mismatch of a gas layer was an effective mean to increase the sound panel insulation. The gas layer $10 \mathrm{~cm}$ thick on the side of the panel produced a substantial increase in the sound insulation in the mid-to-high frequency range. Gasses with a large difference in the impedance maximized the effective sound insulation of a layered system.

An application of the finite element method to different acoustic problems was demonstrated among others by Papadopoulos (2002; 2003), Diaz et al. (2010a; 2010b), АMichi et al. (2010), HÖKMARK (2007), Maluski and Gibbs (2004) and Okuzono et al. (2010). PAPADOPOUlOS $(2002 ; 2003)$ showed that a three-dimensional numerical model of an acoustic laboratory was a useful tool to calculate the sound insulation of different structures. A three-dimensional model was computationally very expensive and could be mainly used to predict the acoustic structure behaviour in a low-frequency band. To overcome this problem, a two-dimensional acoustic laboratory model with a fluid-structure interaction was proposed by DiAz et al. (2010a). A good agreement between experiments and numerical results was achieved for a concrete hollow brick wall. This model was furthermore optimized by Diaz et al. (2010b).

Our research work was motivated by the need to develop composite structural insulated panels CSIPs for residential and light commercial buildings with acoustic properties of the airborne sound insulation meeting standard requirements. The experiments on the sound transmission loss in CSIPs were carried out in a large acoustic test laboratory. Next, numerical simulations of experiments were carried out using a FE coupled acoustic-structural medium model available in the commercial FE package ABAQUS (2010a; 2010b). Steady-state and transient 3D and 2D finite element analyses were performed. Later, several modifications of the CSIPs were proposed based on 2D FE results in order to improve the acoustic properties. The innovative points of our research work are: a) a direct comparison between experimental and numerical results for composite building panels, b) comprehensive comparative $\mathrm{FE}$ analyses (steady state and transient $2 \mathrm{D}$ and $3 \mathrm{D}$ computations) and c) numerical analyses related with modifications CSIPs in order to improve their acoustic properties.

\section{Experiments in acoustic laboratory}

\subsection{Experimental set-up and measurement procedure}

The measurements of the sound reduction index of the wall panel CSIP of Fig. 1 were carried out in the Acoustic Laboratory of Ship Design and Research Centre in Gdańsk (Poland) composed of two reverberation chambers (Fig. 2) with the volume of $212 \mathrm{~m}^{3}$

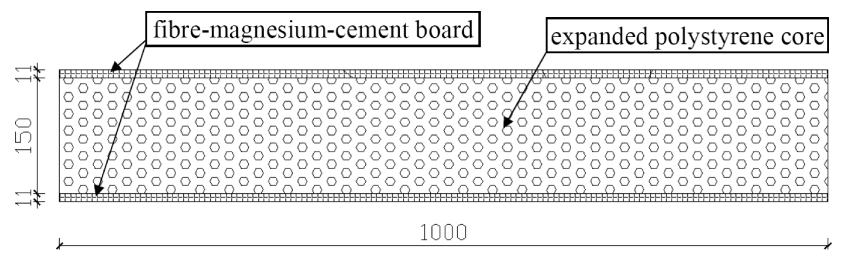

Fig. 1. Geometry of CSIPs (dimensions in [mm]).

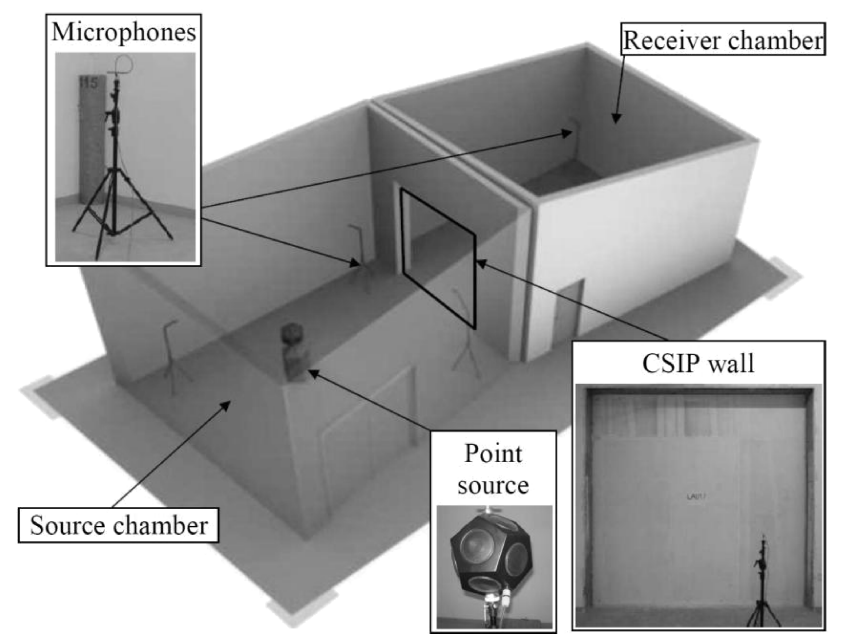

Fig. 2. Acoustic laboratory with measurement equipment. 
and $190 \mathrm{~m}^{3}$, respectively. The proportions between the chamber dimensions ensured a uniform distribution of modal frequencies especially in a low-frequency range. The source chamber was separated from the receiving chamber, and both chambers were separated from the building structure (Fig. 3). In addition, both chambers were installed on the separate foundations. Therefore, the external noise and flanking transmission were reduced to the minimum. The chambers walls were highly sound reflective (they were made from painted concrete). The panel baffle area was about $10 \mathrm{~m}^{2}\left(3.17 \times 3.16 \mathrm{~m}^{2}\right)$. The acoustic baffle was created by CSIPs connected by edge-to-edge to splines from oriented strand boards (Fig. 3). The wood screws were used to connect facesheets and splines on both board sides. The panel was mounted on a steel beam in a floor channel (Fig. 2). All slots needed to insert the connectors were in the core layer.

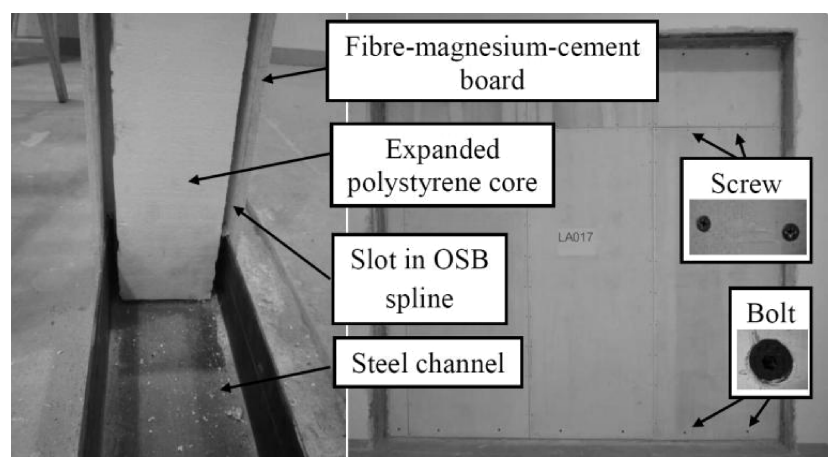

Fig. 3. Tested baffle during construction and connection details.

The airborne sound reduction index $R$ was measured that was the amount of the sound intensity being lost when sound was transmitted from the source to the receiving chamber through a tested baffle. The index $R$ is a function of the frequency $f$ and was calculated as the difference between the average sound pressure in the source and receiving chamber by taking into account the presence of the baffle (ISO 140-3, 1995):

$$
R=L_{1}-L_{2}+10 \times \log \frac{S}{A},
$$

where $L_{1}$ - the average sound pressure level in the source chamber in $[\mathrm{dB}], L_{2}-$ the sound pressure level in the receiving chamber in $[\mathrm{dB}], S$ - the tested sample area in $\left[\mathrm{m}^{2}\right]$ and $A$ - the equivalent absorption area in the receiving chamber in $\left[\mathrm{m}^{2}\right]$. The equivalent absorption area $A$ was calculated as (ISO 140-3, 1995):

$$
A=\frac{0.16 V}{T}
$$

where $V$ is the receiving chamber volume in $\left[\mathrm{m}^{3}\right]$ and $T$ denotes the reverberation time of the receiving chamber in $[\mathrm{s}]$.
The average sound pressure levels $L_{1}$ and $L_{2}$ were given by the following equation:

$$
L=10 \times \log \frac{1}{n} \sum_{i=1}^{n} 10^{L_{i} / 10},
$$

where $n$ denotes the number of test points (stationary microphones) in each chamber and $L_{i}$ is the sound pressure level at the point ' $i$ ' $(\mathrm{dB})$. A sound field was generated by the omnidirectional corner loudspeaker placed opposite to the test-specimen area and emitting the pink (flicker) noise (a signal with a frequency spectrum such that the power spectral density is inversely proportional to the frequency).

Based on the measured curve $R=R(f)$, the weighted sound reduction index $R_{w}$ and the spectrum adaptation terms $C$ and $C_{t r}$ could be calculated in the frequency range of 100-3150 Hz. The terms $C$ and $C_{t r}$ reduce the index $R_{w}$ according to the noise source type (EN-ISO 717-1, 1996):

$$
\begin{aligned}
C & =X_{A 1}-R_{w}, \\
C_{t r} & =X_{A 2}-R_{w},
\end{aligned}
$$

with

$$
X_{A j}=-10 \times \log \sum_{i=1}^{n} 10^{\left(L_{i j}-R_{i}\right) / 10},
$$

where ' $i$ ' is the number of the $1 / 3$ octave band and ' $j$ ' is the number of the adaptation spectrum. Next, the apparent sound reduction indexes $R_{A 1}$ and $R_{A 2}$ (EN-ISO 717-1, 1996) were calculated as:

$$
\begin{aligned}
& R_{A 1}=R_{w}+C, \\
& R_{A 2}=R_{w}+C_{t r} .
\end{aligned}
$$

The index $R_{A 1}$ is applied when the loading noise is the human noise. In turn, the index $R_{A 2}$ is related to the urban traffic noise. The indexes $R_{A 1}, R_{A 2}$ do not take into account a flanking transmission effect. However, the requirements for residential buildings (PN-B-02151-3, 1999) are compared with the apparent sound reduction indexes $R_{A 1}^{\prime}, R_{A 2}^{\prime}$ including the flanking transmission effect. This negative effect can be estimated as $3 \mathrm{~dB}$ for internal walls and $0 \mathrm{~dB}$ for external walls without windows in residential buildings (Szudrowicz et al., 2002).

\subsection{Measurement results}

Figure 4 presents the measured airborne sound insulation curve $R=R(f)$ related to the standard reference curve (ISO $717-1,1996$ ) in the range of the $1 / 3$ octave bandwidth. The frequencies correspond to the octave band centre frequencies. Our CSIP possesses unfortunately two resonant frequencies in the considered frequency band which decrease its sound insulation, namely at $f=630 \mathrm{~Hz}$ and at $f=3150 \mathrm{~Hz}$. 
The symmetric so-called mass-spring-mass resonance at $f=630 \mathrm{~Hz}$ is caused by volume changes of the compressible EPS core between stiff facesheets, and the asymmetric coincidence resonance at $f=3150 \mathrm{~Hz}$ is due to bending waves (VIGRAN, 2008).

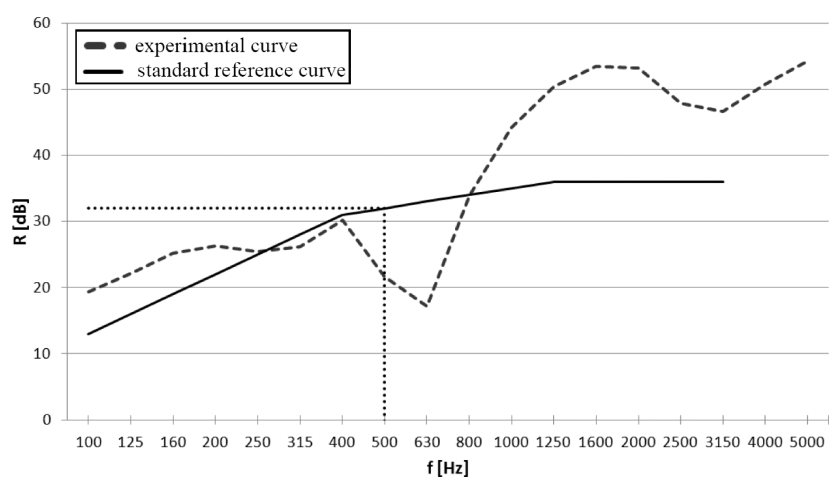

Fig. 4. Sound insulation curve for CSIP from experiments in acoustic laboratory $(R$ - sound reduction index, $f$ - frequency) compared to standard reference curve.

Based on the curve of Fig. 4, the weighted sound reduction index is $R_{w}=32 \mathrm{~dB}$ and the spectrum adaptation terms are $C=-4 \mathrm{~dB}$ and $C_{t r}=-6 \mathrm{~dB}$. In turn, the sound reduction indexes are: $R_{A 1}=28 \mathrm{~dB}$ and $R_{A 1}^{\prime}=25 \mathrm{~dB}$ (internal walls of residential buildings), and $R_{A 2}=26 \mathrm{~dB}$ and $R_{A 2}^{\prime}=26 \mathrm{~dB}$ (external walls of residential buildings loaded with urban traffic noise). According to the polish standard (PN-B-021513, 1999), CSIP does not meet requirements for internal walls in residential buildings (walls in flat rooms in multifamily buildings) by $10 \mathrm{~dB}$. The requirements for external walls are related with the equivalent Aweighted sound level outside buildings. CSIPs do not meet requirements for external walls in residential buildings by $4-7 \mathrm{~dB}$ (the daily and night equivalent A-weighted sound levels are not greater than $60 \mathrm{~dB}$ ). Thus, their airborne sound insulation should be enhanced.

\section{Mathematical model for coupled acoustic-structural medium analyses}

A commercial program ABAQUS (2010a) was used to solve the coupled acoustic-structural problem under transient and steady-state conditions. The acoustic equation was expressed by the equilibrium equation for small motions of a compressible adiabatic fluid with velocity-dependent momentum losses:

$$
\frac{\partial p}{\partial \mathbf{x}}+\gamma \dot{\mathbf{u}}^{f}+\rho_{f} \ddot{\mathbf{u}}^{f}=0
$$

where $p$ - the dynamic fluid pressure, $\mathbf{x}$ - the spatial position of the fluid particle, $\dot{\mathbf{u}}^{f}$ - the fluid particle velocity, $\ddot{\mathbf{u}}^{f}$ - the fluid particle acceleration, $\rho_{f}$ - the fluid mass density and $\gamma$ - the fluid viscosity (volumetric drag). The constitutive fluid behaviour was assumed to be inviscid, linear, and compressible; thus

$$
p=-K_{f} \frac{\partial \mathbf{u}^{f}}{\partial \mathbf{x}}
$$

with $K_{f}$ as the bulk modulus of fluid. By combining Eqs. (7) and (8), one obtained the equation of motion for the fluid in terms of the fluid pressure:

$$
\frac{1}{K_{f}} \ddot{p}+\frac{\gamma}{\rho_{f} K_{f}} \dot{p}-\frac{\partial}{\partial \mathbf{x}}\left(\frac{1}{\rho_{f}} \frac{\partial p}{\partial \mathbf{x}}\right)=0,
$$

The displacements of edge nodes (surface S3 in Fig. 5) were fixed in a third direction. On the surface S2 (Fig. 5), an acoustic-structural interface was applied where the identity of the fluid and solid displacements in a normal direction to the surface was assumed:

$$
\mathbf{n}^{-} \cdot \mathbf{u}^{f}=\mathbf{n}^{-} \cdot \mathbf{u}^{m},
$$

where $\mathbf{u}^{m}$ - the solid displacement, $\mathbf{u}^{f}$ - the fluid displacement and $\mathbf{n}$ - the vector outward normal to the structure.

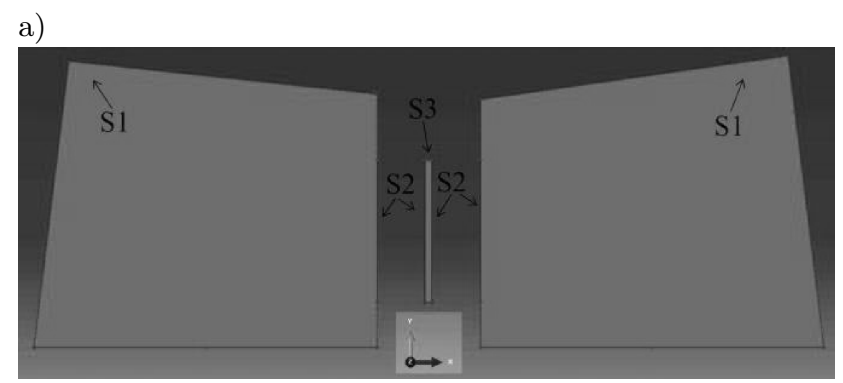

b)

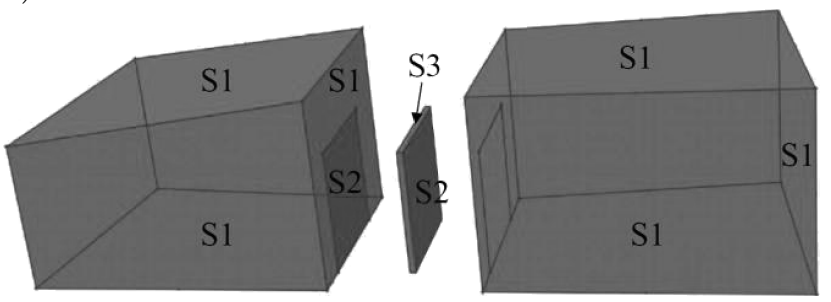

Fig. 5. Boundary conditions used in numerical: a) $2 \mathrm{D}$ and b) 3D model: S1 - impedance boundary conditions, S2 acoustic-structural boundary conditions and S3 - displacements boundary conditions.

The reflection conditions were applied on the surface $\mathrm{S} 1$, by defining the spring $k_{1}$ and the dashpot $c_{1}$ (Fig. 5a). The impedance boundary conditions at any point along the acoustic medium surface were governed by the equation in a transient and steady-state analysis equal to:

$$
\dot{u}_{\text {out }}=\frac{1}{c_{1}} p+\frac{1}{k_{1}} \dot{p}
$$

and

$$
\dot{u}_{\mathrm{out}}=\left(\frac{1}{c_{1}}+i \frac{\Omega}{k_{1}}\right) p=\frac{1}{Z(\Omega)} p,
$$


where $\dot{u}_{\text {out }}$ - the acoustic particle velocity in the outward normal direction of the acoustic medium surface, $p$ - the acoustic pressure, $\dot{p}$ - the time rate of the acoustic pressure, $1 / k_{1}$ - the proportionality coefficient between the pressure and displacement normal to the surface, $1 / c_{1}$ - the proportionality coefficient between the pressure and the velocity normal to the surface, $\Omega$ - the angular frequency and $Z(\Omega)$ - the complex boundary impedance. Equation (11) is in the form of an admittance relation; the impedance expression is simply the inverse. The layer of the material in the admittance form acts as a spring and dashpot in series distributed between the acoustic medium and rigid wall.

The final variational equation for the acoustic medium (equivalent of the virtual work statement for the structure) after taking into account of boundary conditions in the interface was:

$$
\begin{aligned}
& \int_{V f}\left[\delta p\left(\frac{1}{K_{f}} \ddot{p}+\frac{\gamma}{\rho_{f} K_{f}} \dot{p}\right)+\frac{1}{\rho_{f}} \frac{\partial \delta p}{\partial \mathbf{x}} \cdot \frac{\partial p}{\partial \mathbf{x}}\right] \mathrm{d} V \\
& -\int_{S f t} \delta p T_{0} \mathrm{~d} S+\int_{S f r} \delta p\left(\frac{\gamma}{\rho_{f} c_{1}} p+\left(\frac{\gamma}{\rho_{f} k_{1}}+\frac{1}{c_{1}}\right) \dot{p}+\frac{1}{k_{1}} \ddot{p}\right) \mathrm{d} S \\
& +\int_{S f i} \delta p\left(\frac{1}{c_{1}} \dot{p}+\frac{1}{a_{1}} p\right) \mathrm{d} S-\int_{S f s} \delta p \overline{\mathbf{n}} \cdot \ddot{\mathbf{u}}^{m} \mathrm{~d} S+\int_{S f r s} \delta p \\
& \cdot\left(\frac{\gamma}{\rho_{f} c_{1}} p+\left(\frac{\gamma}{\rho_{f} k_{1}}+\frac{1}{c_{1}}\right) \dot{p}+\frac{1}{k_{1}} \ddot{p}-\overline{\mathbf{n}} \cdot \ddot{\mathbf{u}}^{m}\right) \mathrm{d} S=0 .
\end{aligned}
$$

Equation (10) captures the following boundary subregions $S$ :

- $S f t$ - description of the normal derivative of the acoustic pressure per unit density, where $T_{0}$ is the fluid particle acceleration,

- Sfr - the reactive acoustic boundary with a prescribed linear relationship between the fluid acoustic pressure and its normal derivative,

- $S f i$ - the radiating acoustic boundary, where $a_{1}$ is the impedance parameter,

- $S f s$ - the boundary, where the motion of an acoustic medium is directly coupled to the solid motion (the acoustic and structural media have the same displacements normal to the boundary, but the tangential ones are uncoupled,

- Sfrs - the acoustic-structural boundary, where displacements are linearly coupled but not necessarily identical due to the presence of a reactive intervening layer (the layer induces an impedance condition between the relative normal velocity between the acoustic fluid, solid structure and acoustic pressure).
In turn, the structural behaviour was defined by the virtual work equation:

$$
\begin{array}{r}
\int_{V} \delta \varepsilon: \boldsymbol{\sigma} \mathrm{d} V+\int_{V} \rho \delta \mathbf{u} \cdot \ddot{\mathbf{u}} \mathrm{d} V+\int_{V} \rho \alpha_{c} \delta \mathbf{u} \cdot \dot{\mathbf{u}} \mathrm{d} V \\
+\int_{S} p \delta \mathbf{u} \cdot \mathbf{n} \mathrm{d} S+\int_{S} \delta \mathbf{u} \cdot \mathbf{t} \mathrm{d} S=0
\end{array}
$$

where $\dot{\mathbf{u}}$ and $\ddot{\mathbf{u}}-$ the velocity and the acceleration, respectively, $p$ - the pressure acting on the fluidstructural interface $\rho$ - the mass density, $\alpha_{c}$ - the damping factor (part of the Rayleigh damping proportional to the mass), $\boldsymbol{\sigma}$ - the stress tensor, $\mathbf{n}-$ the outward normal to the structure, $\mathbf{t}$ - the surface traction applied to the structure and $\delta \varepsilon$ and $\delta \mathbf{u}-$ the strain and displacement variations compatible with $\delta \mathbf{u}$, respectively.

Equations (13) and (14) define a variational timedependent problem for the coupled fields $\mathbf{u}$ and $p$. To solve the sound pressure variation in the time domain, the transient dynamic equations were integrated through time using an explicit dynamic integration method. To determine a signal in the frequency domain, the fast Fourier transform was used.

In the case of a steady-state linear dynamic analysis, it was assumed that the structure was subjected to continuous harmonic excitations. All model degrees of freedom and loads were assumed to vary harmonically at the angular frequency $\Omega$ :

$$
\begin{gathered}
f=\bar{f} \exp i \Omega t, \quad \dot{f}=i \Omega f, \\
\text { and } \quad \ddot{f}=i \Omega \dot{f},
\end{gathered}
$$

where $\bar{f}$ is the constant complex amplitude of the variable $f$. Introducing Eq. (15) into Eqs. (13) and (14), a time-independent problem had to be solved. A steadystate analysis resulted in a fixed amplitude as a response to harmonic loads at a particular frequency. Equations (8) and (9) became

$$
\Omega^{2} \frac{1}{K_{f}} \widetilde{p}-\frac{\partial}{\partial \mathbf{x}} \cdot\left(\frac{1}{\widehat{\rho}} \frac{\partial \widetilde{p}}{\partial \mathbf{x}}\right)=0 .
$$

The final variational equation is

$$
\begin{aligned}
& \int_{V f}\left[-\Omega^{2} \delta p\left(\frac{1}{K_{f}} \tilde{p}\right)+\frac{1}{\widetilde{\rho}} \frac{\partial \delta p}{\partial \mathbf{x}} \cdot \frac{\partial \widetilde{p}}{\partial \mathbf{x}}\right] \mathrm{d} V-\int_{S f t}-\delta p \cdot a_{\text {in }} \mathrm{d} S \\
& +\int_{S f r \cup S f i} \delta p\left(\frac{i \Omega}{c_{1}}-\frac{\Omega^{2}}{k_{1}}\right) \widetilde{p} \mathrm{~d} S+\int_{S f s} \delta p \Omega^{2} \overline{\mathbf{n}} \cdot \widetilde{\mathbf{u}}^{m} \mathrm{~d} S \\
& +\int_{S f r s} \delta p\left(\frac{i \Omega}{c_{1}} \widetilde{p}-\frac{\Omega^{2}}{k_{1}} \widetilde{p}+\Omega^{2} \overline{\mathbf{n}} \cdot \widetilde{\mathbf{u}}^{m}\right) \mathrm{d} S=0
\end{aligned}
$$

where $a_{\text {in }}$ - the fluid particle inward acceleration, $\widetilde{p}-$ the constant complex amplitude of the acoustic pres- 
sure, $\widetilde{\rho}$ - the constant complex amplitude of the acoustic density, $\widetilde{\mathbf{u}}-$ the constant complex amplitude of the structure displacement.

\section{Input FE data}

In the first research step, in order to reduce the computation time, a simplified two-dimensional numerical model was used (Figs. 5a and 6). The model's geometry was the exact representation of the real acoustic laboratory test chambers (Fig. 2). The areas of the source and receiver chamber were $37.9 \mathrm{~m}^{2}$ and $38.8 \mathrm{~m}^{2}$, respectively. The following distance requirements for the acoustic laboratory test chambers (ISO 140-3, 1995) related to source and microphones positions were taken into account:

- the minimum distance of $0.7 \mathrm{~m}$ from the measurement points to the chamber baffle,

- the minimum distance of $0.7 \mathrm{~m}$ between the measurement points,

- the distance more than $1 \mathrm{~m}$ between the measurement points and sound source,

- the distance more than $1 \mathrm{~m}$ between the measurement points and baffle.

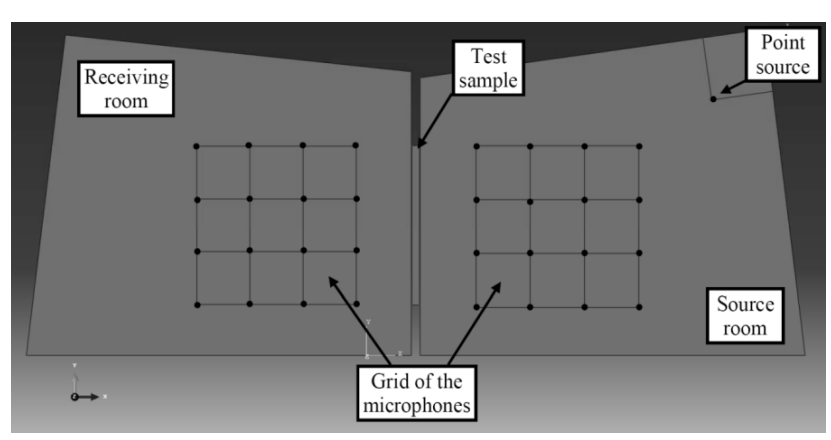

Fig. 6. Two-dimensional numerical FE model of acoustic laboratory of Fig. 2 (grid visualizes microphones' distribution in both acoustic chambers).

The structural flanking transmission was neglected. Since all surfaces of chambers were highly reflective, the absorption coefficient of 0.05 was chosen (for the painted concrete wall), which remained constant in the entire frequency range. A statistical absorption factor theory (VIGRAN, 2008) was used to determine the spring $k_{1}$ and dashpot $c_{1}$ parameters. The statistical absorption factor $\alpha_{\text {stat }}$ was:

$$
\alpha_{\text {stat }}=\int_{0}^{\pi / 2}\left[1-\left|R_{p}\right|^{2}\right] \sin \varphi \cos \varphi \mathrm{d} \varphi,
$$

where $R_{p}$ is the complex pressure reflection factor, $\varphi$ is the angle between the incident wave and normal to the surface. The factor $R_{p}$ is related with the boundary surface impedance and impedance of an acoustic medium. It means that the sound absorption factor, boundary impedance, the spring $k_{1}$ and dashpot $c_{1}$ parameters are associated with each other (Eqs. (12) and (18)). The parameters were assumed as follows $\left(\alpha_{\text {stat }}=0.05\right): 1 / k_{1}=0$ (imaginary part of admittance) and $1 / c_{1}=1.6 \times 10^{-5}$ (real part of admittance).

The acoustic medium was modelled as a compressible, inviscid and linear fluid with the following parameters: bulk density $\rho=1.2 \mathrm{~kg} / \mathrm{m}^{3}$ and bulk modulus $K=142 \mathrm{kPa}$ (PAPAdopoulos, 2003). The sound source point with the amplitude of $2 \mathrm{~Pa}$ was located in the upper right corner. The acoustic load with the magnitude $100 \mathrm{~dB}$ may cause stresses located in an elastic region of the test panel baffle. The density values $\rho$ of the panel layers were determined experimentally (Smakosz et al., 2012; Smakosz, Tejchman, 2014) (Table 1). The material properties (Young's modulus $E$, Poisson's $\nu$ ratio, loss factor $\eta$ ) were based on the literature data (Xenaki, 2005; Cremer, HECKL, 1998) and own static strength experiments with fibre-magnesium-cement boards (SMAKosz et al., 2012; Smakosz, Tejchman, 2014) (Table 1).

Table 1. Material properties adopted in numerical calculations.

\begin{tabular}{|l|c|c|c|c|}
\hline Component & $\begin{array}{c}\text { Young's } \\
\text { modulus } \\
E \\
{[\mathrm{MPa}]}\end{array}$ & $\begin{array}{c}\text { Poisson's } \\
\text { ratio } \\
\nu \\
{[-]}\end{array}$ & $\begin{array}{c}\text { Mass } \\
\text { density } \\
\rho \\
{\left[\mathrm{kg} / \mathrm{m}^{3}\right]}\end{array}$ & $\begin{array}{c}\text { Loss } \\
\text { factor } \\
\eta \\
{[-]}\end{array}$ \\
\hline $\begin{array}{l}\text { fibre- } \\
\text { magnesium- } \\
\text { cement board }\end{array}$ & 5000 & 0.18 & 1100 & 0.01 \\
\hline $\begin{array}{l}\text { expanded } \\
\text { polystyrene } \\
\text { core }\end{array}$ & 13.7 & 0.29 & 21 & 0.05 \\
\hline
\end{tabular}

According to Xenaki (2005), the dynamic elastic properties of expanded polystyrene foams can be estimated as (with $E=2 G(1+\nu)$ ):

$$
G=0.32 \rho-1.4 \quad \text { and } \quad \nu=0.22+0.0033 \rho .
$$

In steady-state FE analyses, the second-order twodimensional 8-noded quadrilateral elements (AC2D8) with pressure as the degree of freedom were used to simulate an acoustic medium. In transient analyses, the first-order elements (AC2D4R) were chosen. The solid specimen was spatially discretized by planestrain, first-order 4-node quadrilateral elements with displacement degrees of freedom (with the reduced integration and hourglass control). At least six nodes per the wavelength in the acoustic medium were chosen. The maximum inter-nodal interval of finite elements was:

$$
D_{\max }<\frac{c}{n_{\min } f_{\max }},
$$


where $D_{\max }$ - the maximum inter-nodal interval in the element of the mesh, $c$ - the sound speed, $n_{\min }-$ the minimum number of the inter-nodal intervals per acoustic wavelength (minimum 6 and 3 for the firstand second-order elements, respectively) and $f_{\max }-$ the maximum frequency excitation in the medium. Based on preliminary calculations, the mesh element dimension describing the acoustic medium in a $2 \mathrm{D}$ steady state analysis was mainly $40 \mathrm{~mm}$. The magnesium board and the polystyrene core were always modelled by $10 \mathrm{~mm}$ large quads, while the mesh quad size of the glass wool was $0.6 \mathrm{~mm}$. In turn, the acoustic medium in $2 \mathrm{D}$ transient computations was modelled by elements of $10 \mathrm{~mm}$. The magnesium board and polystyrene core were always described by $5 \mathrm{~mm}$ large quadrilaterals.

During 3D steady-state simulations, an acoustic medium was modelled by the second-order cubic elements of $160 \mathrm{~mm}$, the magnesium board was described by the second-order shell elements of $80 \mathrm{~mm}$ and the polystyrene core by the second-order cubic elements of $80 \mathrm{~mm}$ (with the reduced integration). In 3D transient analyses, the first-order cubic elements of $40 \mathrm{~mm}$ were used to describe an acoustic medium, first-order shell elements of $40 \mathrm{~mm}$ to model magnesium boards and first-order cubic elements of $40 \mathrm{~mm}$ to describe a polystyrene core (with the full integration).

The sound pressure level was computed based on the nodal pressures:

$$
L_{p}=10 \times \log \left(\frac{p_{\mathrm{rms}}^{2}}{p_{\mathrm{ref}}^{2}}\right),
$$

where $p_{\text {rms }}$ - the root mean square pressure and $p_{\text {ref }}$ - the reference pressure $(20 \mu \mathrm{Pa})$. The average sound pressure level in the $1 / 3$ octave band was calculated at each microphone position (16 points in each chamber) as (PApAdopoulos, 2003):

$$
L_{i}=10 \times \log \left(\frac{\int_{f_{i}}^{f_{i+1}} 10^{L(f) / 10} \mathrm{~d} f}{f_{i+1}-f_{i}}\right),
$$

where $L(f)$ - the function of the sound pressure level in the frequency domain, $f_{i+1}$ - the top of the $i$-th frequency band and $f_{i}$ - the bottom of the $i$-th frequency band. The discrete signal $L(f)$ was used in the post-processing computation. In steady-state calculations, the excitation frequencies with the $1 \mathrm{~Hz}$ interval from each $1 / 3$ octave band were chosen to calculate $L_{i}$ in Eq. (22), and 5524 frequencies were taken for each microphone position within the entire frequency band. During transient analyses, the time dependent data were transformed to the frequency dependent data by the fast Fourier transform. All excitation frequencies (from $89 \mathrm{~Hz}$ up to $5612 \mathrm{~Hz}$ ) with the $1 \mathrm{~Hz}$ interval were chosen. The analyzed time period was equal to $5 \mathrm{~s}$.

In order to achieve stability and convergence in explicit dynamic analyses the time increment size $\Delta t$ satisfied the condition (ABAQUS, 2010a):

$$
\Delta t \leq \min \left(\frac{L_{e}}{c_{d}}\right),
$$

where $L_{e}$ - the characteristic element dimension and $c_{d}$ - the wave speed in the material:

$$
c_{d} \leq \sqrt{\frac{(\tilde{\lambda}+2 \widetilde{\mu})}{\rho}},
$$

where $\widetilde{\lambda}$ and $\widetilde{\mu}$ are the effective Lame's constants and $\rho$ is the density of the material. The stable time increment was equal to $1.5 \cdot 10^{-6} \mathrm{~s}$ throughout the study. Adhesive layers and spline connections were neglected in numerical analyses.

The 2D calculations were performed using the PC computer $4 \mathrm{CPU} 2.7 \mathrm{GHz}$ (8 GB RAM memory). The total computational average time for the model was 10 hours for a $2 \mathrm{D}$ steady state analysis (acoustic medium was modelled by second-order elements of $40 \mathrm{~mm}$ ) and 45 hours for an 2D transient explicit dynamic analysis (acoustic medium was simulated by first-order elements of $10 \mathrm{~mm}$, and CSIP was described by first-order $5 \mathrm{~mm}$ elements). In turn, the 3D steady-state calculations were performed using PC $32 \mathrm{CPU} 2.3 \mathrm{GHz}$ and the 3D transient calculations using $\mathrm{PC} 8 \mathrm{CPU}$ $2.3 \mathrm{GHz}$. The 3D computations lasted about 26 days (steady-state approach) and 8 days (transient approach).

\section{FE results}

The calculated sound insulation curves for a steady-state and a transient approach are shown in

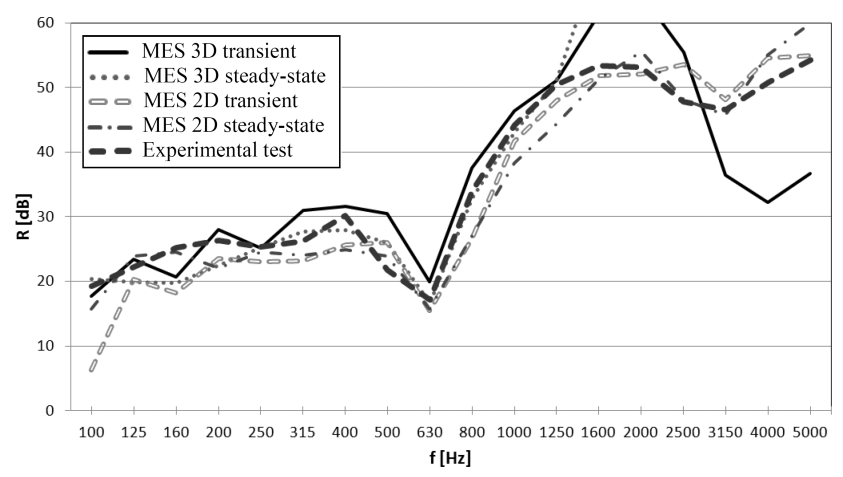

Fig. 7. Sound insulation curve for CSIP from experiments and FE simulations ( $R$ - sound reduction index, $f-$ frequency). 
Fig. 7 as compared to the experimental curve of Fig. 4 . The measured and calculated sound insulation ratings from 2D simulations are summarized in Table 2. A comparison in Table 2 was not performed with 3D results because our 3D model with the assumed mesh size was not able to properly describe the acoustic behaviour in the medium and high frequency range of $f>1250 \mathrm{~Hz}$ (Fig. 7).

Table 2. Measured and calculated weighted sound reduction index, spectrum adaptation terms and apparent sound reduction indexes for external walls in residential buildings.

\begin{tabular}{|l|c|c|c|c|l|}
\hline \multicolumn{1}{|c|}{ Ratings } & $\begin{array}{c}R_{w} \\
{[\mathrm{~dB}]}\end{array}$ & $\begin{array}{c}C \\
{[\mathrm{~dB}]}\end{array}$ & $\begin{array}{c}C_{t r} \\
{[\mathrm{~dB}]}\end{array}$ & $\begin{array}{c}R_{A 1} \\
{[\mathrm{~dB}]}\end{array}$ & $\begin{array}{l}R_{A 2} \\
{[\mathrm{~dB}]}\end{array}$ \\
\hline measurements & 32 & -4.4 & -6 & 27.5 & 26 \\
\hline $\begin{array}{l}\text { steady-state 2D } \\
\text { FE analyses }\end{array}$ & 29 & -2.9 & -4.6 & 26.1 & 24.4 \\
\hline $\begin{array}{l}\text { transient 2D FE } \\
\text { analyses }\end{array}$ & 29 & -3.4 & -6.8 & 25.6 & 22.2 \\
\hline
\end{tabular}

The experimental and 2D numerical curves are very similar. However, a better agreement with the experiment was obtained by means of a steady-state approach. The transient $2 \mathrm{D}$ analysis results indicated a clear discrepancy at $f=100 \mathrm{~Hz}$. The measured two resonant frequencies were reflected in the $2 \mathrm{D}$ numerical curves $\left(f_{1}=630 \mathrm{~Hz}\right.$ and $\left.f_{2}=3150 \mathrm{~Hz}\right)$. Both the $3 \mathrm{D}$ steady-state and transient results were similar. An excellent agreement between the experimental results and $3 \mathrm{D}$ results was achieved in a medium frequency range $<1250 \mathrm{~Hz}$ (with some small differences in a low frequency range at $f<250 \mathrm{~Hz}$ ).

The sound ratings obtained from measurements and numerical steady-state 2D simulations (Table 2) are almost the same. There is no difference in the weighted sound reduction index $R_{w}$ and the discrepancies in the $R_{A 1}, R_{A 2}, C$ and $C_{t r}$ indexes are solely $1 \mathrm{~dB}$. In turn, the differences between measurements and transient simulation $2 \mathrm{D}$ results are more pronounced: about $2 \mathrm{~dB}$ for $R_{w}$ and about $4 \mathrm{~dB}$ for $R_{A i}$ (caused by discrepancies at $f<100 \mathrm{~Hz}$, Fig. 7).

The experimental and numerical results of Fig. 7 were also compared with the empirical curve proposed by KRAKERS (2009):

$$
\begin{aligned}
R= & R_{0}-10 \log _{10}\left[\frac{2 \sigma_{\text {radd }}}{\left(1-\left(\frac{f_{11}}{f}\right)^{2}\right)^{2}\left(1-\left(\frac{f}{f_{c}}\right)^{2}\right)^{2}+\eta_{e q}^{2}}+\frac{\pi \sigma^{2} f_{c}}{2 \eta_{c} f}\right] \\
& +10 \log _{10}\left[\left(1-\left(\frac{f}{f_{\text {dil }}}\right)^{2}\right)^{2}+\eta_{e q}^{2}\right],
\end{aligned}
$$

where $R_{0}$ denotes the sound insulation determined by the mass law $[\mathrm{dB}]$ :

$$
R_{0}=20 \log _{10} m+20 \log _{10} f-42,
$$

with $m$ as the sandwich surface mass ( $f$ - frequency) and $f_{\text {dil }}$ as the symmetric resonant frequency $\left(f_{\text {dil }}=\right.$ $450 \mathrm{~Hz})$ :

$$
f_{\text {dil }}=\frac{1}{2 \pi}\left[\frac{2 K_{c}}{h_{2}\left(\rho_{1} h_{1}+\rho_{2} h_{2} / 6\right)}\right]^{1 / 2},
$$

with

$$
K_{c}=\frac{E_{2}\left(1-\nu_{2}\right)}{\left(1-2 \nu_{2}\right)\left(1+\nu_{2}\right)},
$$

where $h_{1}$ - the facesheet thickness $\left(h_{1}=11 \mathrm{~mm}\right), \rho_{1}$ - the facesheet mass density $\left(\rho_{1}=1100 \mathrm{~kg} / \mathrm{m}^{3}\right), h_{2}$ - the core thickness $\left(h_{2}=150 \mathrm{~mm}\right), \rho_{2}-$ core mass density $\left(\rho_{2}=21 \mathrm{~kg} / \mathrm{m}^{3}\right), \nu_{2}$ - the core Poisson's ratio $\left(\nu_{2}=0.29\right)$ and $E_{2}-$ the core Young's modulus $\left(E_{2}=\right.$ $13.7 \mathrm{GPa}$, Table 1 ) and $f_{c}$ - the critical frequency $f_{c}=$ $3080 \mathrm{~Hz}$ calculated as:

$$
f_{c}=\frac{c_{0}^{2}}{2 \pi} \sqrt{\frac{m}{D}},
$$

where $D$ is the sandwich bending stiffness per the unit width and $c_{0}$ is the sound speed in air $\left(c_{0}=340 \mathrm{~m} / \mathrm{s}\right)$. The parameter $f_{11}$ in Eq. (25) denotes the first natural frequency of a rectangular panel with the dimensions $l_{x}$ and $l_{y}\left(f_{11}=48 \mathrm{~Hz}\right.$ and $\left.l_{x}=l_{x}=3.16 \mathrm{~m}\right)$ :

$$
f_{11}=\frac{\pi}{2} \sqrt{\frac{D}{m}}\left[\left(\frac{1}{l_{x}}\right)^{2}+\left(\frac{1}{l_{y}}\right)^{2}\right] .
$$

The coefficients $\sigma_{\text {rad }}$ and $\sigma_{\text {radd }}$ are the radiation coefficients:

$$
\sigma_{\mathrm{rad}}=\left\{\begin{array}{cl}
\frac{U c_{0}}{\pi^{2} S f_{c}^{3 / 2}} \sqrt{f} & f \ll f_{c} \\
0.45 \sqrt{\frac{U f_{c}}{c_{0}}} & f=f_{c} \\
1 & f \gg f_{c}
\end{array}\right.
$$

and

$$
\sigma_{\text {radd }} \cong \frac{1}{2}\left(0.2+\ln \left(2 \pi \frac{f}{c_{0}} \sqrt{S}\right)\right),
$$

where $U$ is the panel perimeter $(U=12.6 \mathrm{~m}), S$ is the panel area $\left(S=10 \mathrm{~m}^{2}\right), \eta_{c}$ denotes the core loss factor $\left(\eta_{c}=0.05\right)$ and $\eta_{e q}$ is the equivalent loss factor $\left(\eta_{e q}=0.087\right)$ :

$$
\eta_{\mathrm{eq}} \cong \sqrt{\eta_{c}^{2}+0.1 \eta_{c}}
$$

The analytical sound insulation curve (Fig. 8) shows a good match with the experiment in a low and medium frequency range, but large differences occur in a high frequency range $(f>1600 \mathrm{~Hz})$. The analytical and experimental sound insulation index $R$ at the symmetric resonance frequency $(f=630 \mathrm{~Hz})$ are different. This causes the discrepancy of about $4 \mathrm{~dB}$ between the experimental and analytical sound reduction index $R_{A}$. 


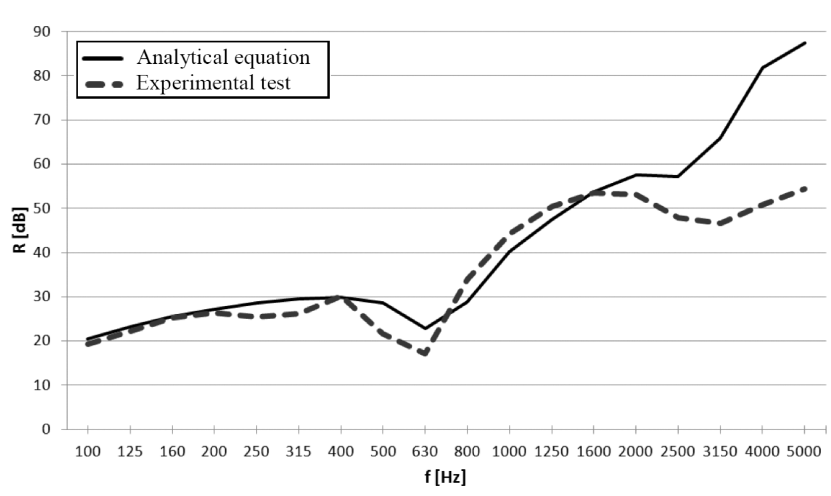

Fig. 8. Sound insulation curve for CSIP from experiments and analytical formula by KraKers (2009) $(R$ - sound reduction index, $f$ - frequency).

The dependence of the sound insulation curve on the mesh size in a steady-state $2 \mathrm{D}$ analysis is shown in Fig. 9. The mesh size of second-order quadrilateral elements of the acoustic medium was 10, 20, 40 and $80 \mathrm{~mm}$. In turn, the mesh size of the wall panel was always $10 \mathrm{~mm}$ (second-order elements with the reduced integration). For the finest FE mesh of about 450'000 finite elements were used in each chamber. The results presented in Fig. 9 show that during steady-state analyses even larger quadrilateral elements (with the size of $40 \mathrm{~mm}$ ) might be used.

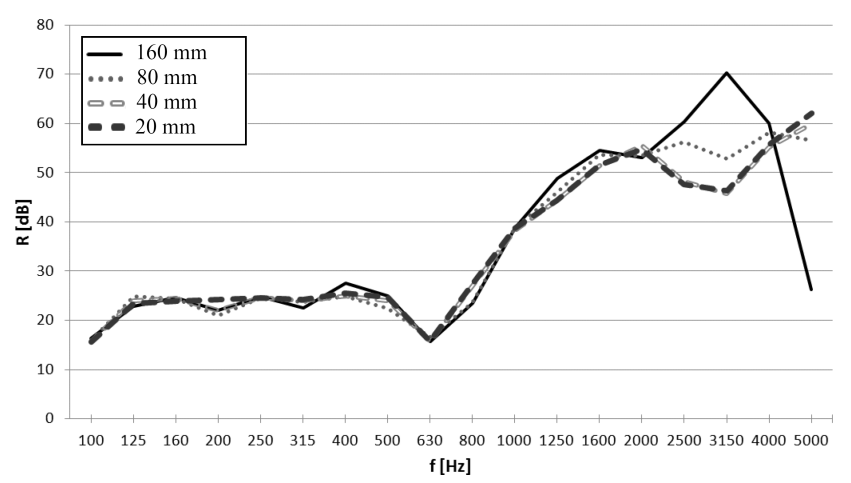

Fig. 9. Sound insulation curve for CSIP from FE steadystate analyses with different mesh size of acoustic medium $(R$ - sound reduction index, $f$ - frequency).

\section{Improvement of sound insulation of CSIPs}

Several modifications of CSIPs were numerically tested in steady-state 2D FE analyses to improve its sound insulation (Subsec. 2.3) (Fig. 10). Two type modifications were considered: a) glass wool layer, air void insert and glass wool insert were put symmetrically against the horizontal axis or b) two CSIPs were mutually connected through a glass wool layer (Table 3, Fig. 10).

The geometry of air voids and the glass wool insert was the same. The following material parameters were assumed for the glass wool: $\rho=101 \mathrm{~kg} / \mathrm{m}^{3}$, $E=303 \mathrm{kPa}, \nu=0$ and loss factor $\eta=0.05$ (NAOKI,

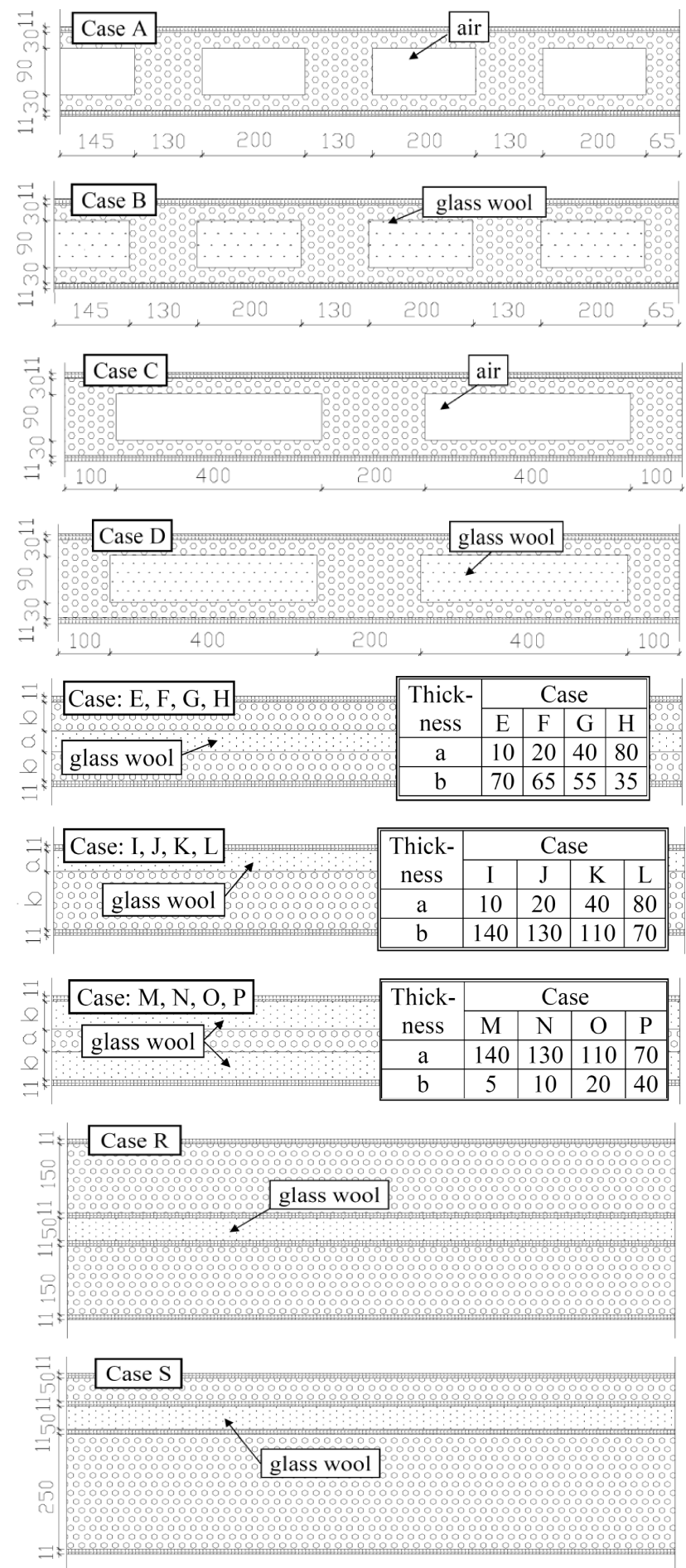

Fig. 10. CSIP with different modifications 'A'-'S' of Table 3 (dimensions in millimetres).

TAKAYASU, 2007; TARNOW, 2005) and for the air layer: $\rho=1.2 \mathrm{~kg} / \mathrm{m}^{3}$ and $K=142 \mathrm{kPa}$ (PAPAdopoulos, 2003). The second-order elements of $40 \mathrm{~mm}$ were used for the acoustic medium. The influence of the different CSIP modifications listed in Table 3 on the sound insulation curve is shown in Figs. 11-18. In turn, Table 4 summarizes the results of the calculated sound insulation ratings $R_{w}, C, C_{t r}, R_{A 1}$ and $R_{A 2}$. 
Table 3. CSIPs with different modifications for FE analyses (Fig. 10).

\begin{tabular}{|c|c|c|}
\hline Modification case (see Fig. 10) & Modification type & Size and location modified component \\
\hline 'A' & Air voids & Area $200 \times 90 \mathrm{~mm}^{2}$, spacing $265 \mathrm{~mm}$ \\
\hline 'B' & Glass wool insert & Area $200 \times 90 \mathrm{~mm}^{2}$, spacing $265 \mathrm{~mm}$ \\
\hline 'C' & Air voids & Area $400 \times 90 \mathrm{~mm}^{2}$, spacing $500 \mathrm{~mm}$ \\
\hline 'D' & Glass wool insert & Area $400 \times 90 \mathrm{~mm}^{2}$, spacing $500 \mathrm{~mm}$ \\
\hline 'E' & Glass wool layer & Continuous glass wool layer (thickness $10 \mathrm{~mm}$ ) \\
\hline 'F' & Glass wool layer & Continuous glass wool layer (thickness $20 \mathrm{~mm}$ ) \\
\hline 'G' & Glass wool layer & Continuous glass wool layer (thickness $40 \mathrm{~mm}$ ) \\
\hline 'H' & Glass wool layer & Continuous glass wool layer (thickness $80 \mathrm{~mm}$ ) \\
\hline 'I' & Glass wool layer & Continuous glass wool layer (thickness $10 \mathrm{~mm}$ ) \\
\hline 'J' & Glass wool layer & Continuous glass wool layer (thickness $20 \mathrm{~mm}$ ) \\
\hline ' $\mathrm{K}$ ' & Glass wool layer & Continuous glass wool layer (thickness $40 \mathrm{~mm}$ ) \\
\hline 'L' & Glass wool layer & Continuous glass wool layer (thickness $80 \mathrm{~mm}$ ) \\
\hline 'M' & Glass wool layer & $\begin{array}{l}\text { Continuous double glass wool layer } \\
\text { (thickness of the single layer } 5 \mathrm{~mm} \text { ) }\end{array}$ \\
\hline ' $\mathrm{N}$ ' & Glass wool layer & $\begin{array}{l}\text { Continuous double glass wool layer } \\
\text { (thickness of the single layer } 10 \mathrm{~mm} \text { ) }\end{array}$ \\
\hline ' $\mathrm{O}^{\prime}$ & Glass wool layer & $\begin{array}{l}\text { Continuous double glass wool layer } \\
\text { (thickness of the single layer } 20 \mathrm{~mm} \text { ) }\end{array}$ \\
\hline 'P' & Glass wool layer & $\begin{array}{l}\text { Continuous double glass wool layer } \\
\text { (thickness of the single layer } 40 \mathrm{~mm} \text { ) }\end{array}$ \\
\hline ' $\mathrm{R}$ ' & Double CSIP & $\begin{array}{l}\text { Continuous glass wool layer (thickness } 50 \mathrm{~mm} \text { ) } \\
\text { between two identical CSIPs }\end{array}$ \\
\hline 'S' & Double CSIP & $\begin{array}{l}\text { Continuous glass wool layer (thickness } 50 \mathrm{~mm} \text { ) } \\
\text { between two different CSIPs }\end{array}$ \\
\hline
\end{tabular}

Table 4. Effect of modifications on sound insulation parameters of CSIPs from FE studies.

\begin{tabular}{|l|c|c|c|c|c|}
\hline Modification case & $\begin{array}{c}R_{w} \\
{[\mathrm{~dB}]}\end{array}$ & $\begin{array}{c}C \\
{[\mathrm{~dB}]}\end{array}$ & $\begin{array}{c}C_{t r} \\
{[\mathrm{~dB}]}\end{array}$ & $\begin{array}{c}R_{A 1} \\
{[\mathrm{~dB}]}\end{array}$ & $\begin{array}{c}R_{A 2} \\
{[\mathrm{~dB}]}\end{array}$ \\
\hline No modification & 29 & -2.9 & -4.6 & 26.1 & 25.1 \\
\hline Modification case 'A' & 29 & -1.7 & -4.6 & 27.3 & 24.4 \\
\hline Modification case 'B' & 33 & -2.6 & -6.0 & 30.4 & 27.0 \\
\hline Modification case 'C' & 32 & -2.5 & -6.9 & 29.5 & 25.1 \\
\hline Modification case 'D' & 35 & -2.8 & -8.1 & 32.2 & 26.9 \\
\hline Modification case 'E' & 34 & -3.3 & -7.1 & 30.7 & 26.9 \\
\hline Modification case 'F' & 34 & -3.2 & -7.7 & 30.8 & 26.3 \\
\hline Modification case 'G' & 37 & -5.2 & -10.4 & 31.8 & 26.6 \\
\hline Modification case 'H' & 36 & -3.9 & -10.7 & 32.1 & 25.3 \\
\hline Modification case 'I' & 33 & -2.4 & -6.1 & 30.6 & 26.9 \\
\hline Modification case 'J' & 35 & -4.0 & -8.7 & 31.0 & 26.3 \\
\hline Modification case 'K' & 37 & -5.2 & -10.6 & 31.8 & 26.4 \\
\hline Modification case 'L' & 37 & -4.6 & -11.5 & 32.4 & 25.5 \\
\hline Modification case 'M' & 33 & -3.2 & -6.8 & 29.8 & 26.2 \\
\hline Modification case 'N' & 34 & -4.1 & -8.7 & 29.9 & 25.3 \\
\hline Modification case 'O' & 35 & -4.4 & -9.6 & 30.6 & 25.4 \\
\hline Modification case 'P' & 35 & -3.4 & -10.1 & 31.6 & 24.9 \\
\hline Modification case 'R' & 34 & -4.0 & -7.5 & 30.0 & 26.5 \\
\hline Modification case 'S' & 40 & -3.9 & -11.6 & 36.1 & 28.4 \\
\hline
\end{tabular}




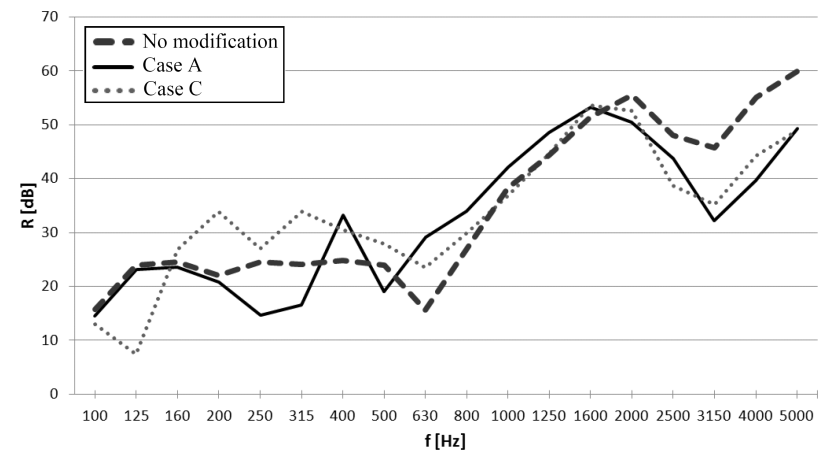

Fig. 11. Sound insulation curve for modified CSIP from FEM: influence of air void inserts from Table $3(R$ - sound reduction index, $f$ - frequency).

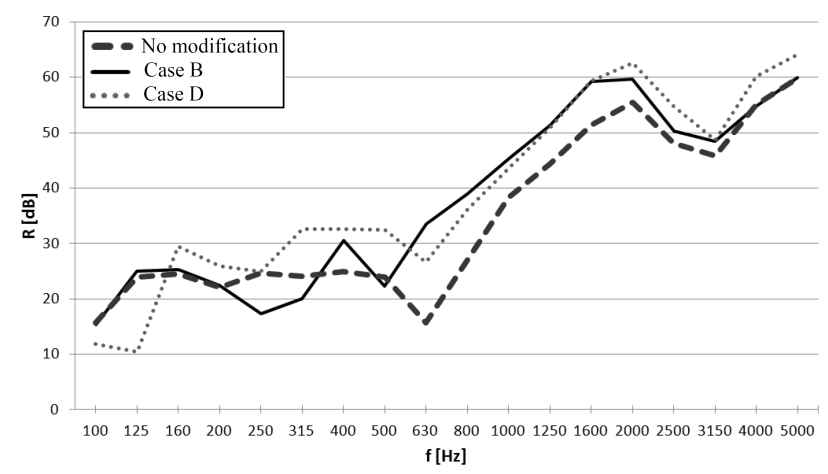

Fig. 12. Sound insulation curve for modified CSIP from FEM: influence of glass wool inserts from Table $3(R-$ sound reduction index, $f$ - frequency).

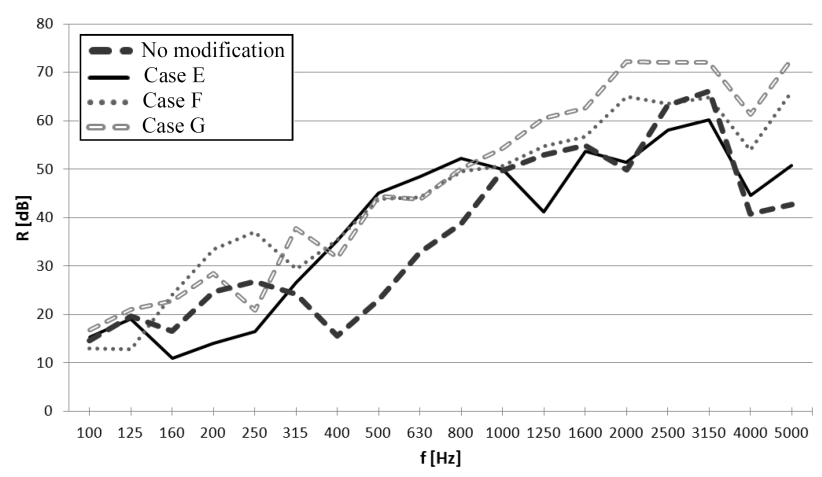

Fig. 13. Sound insulation curve for modified CSIP from FEM: influence of glass wool layer from Table $3(R-$ sound reduction index, $f$ - frequency).

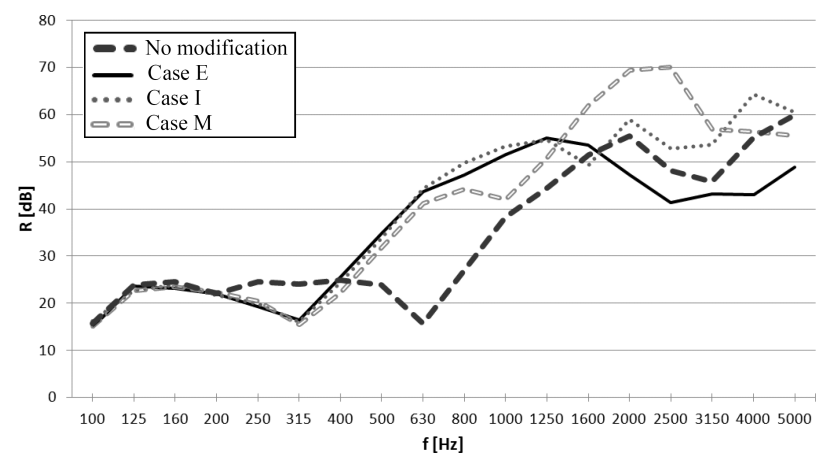

Fig. 14. Sound insulation curve for modified CSIP from FEM: influence of glass wool layer position from Table 3 $(R$ - sound reduction index, $f$ - frequency).

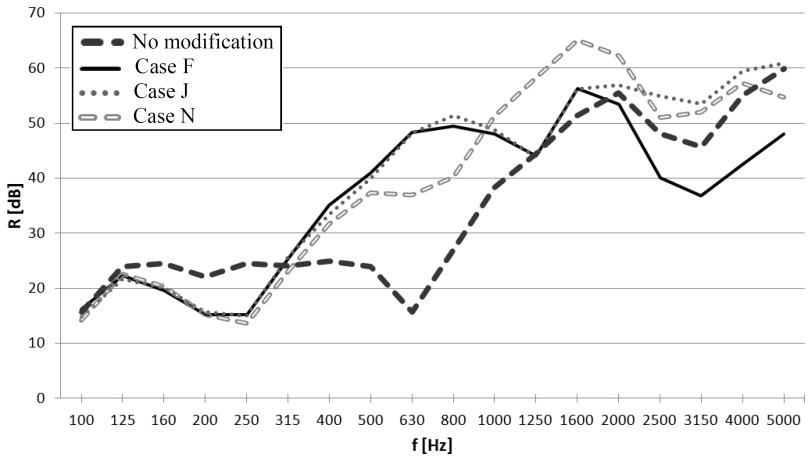

Fig. 15. Sound insulation curve for modified CSIP from FEM: influence of glass wool layer position from Table 3 ( $R$ - sound reduction index, $f$ - frequency).

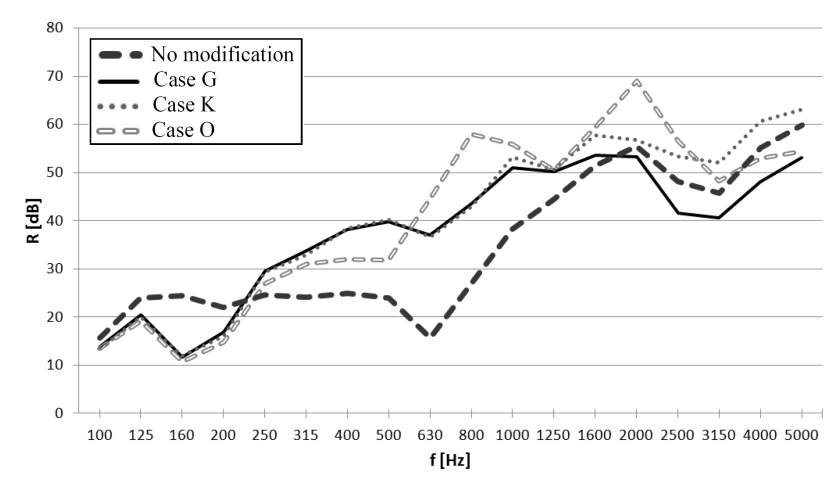

Fig. 16. Sound insulation curve for modified CSIP from FEM: influence of glass wool layer position from Table 3 ( $R$ - sound reduction index, $f$ - frequency).

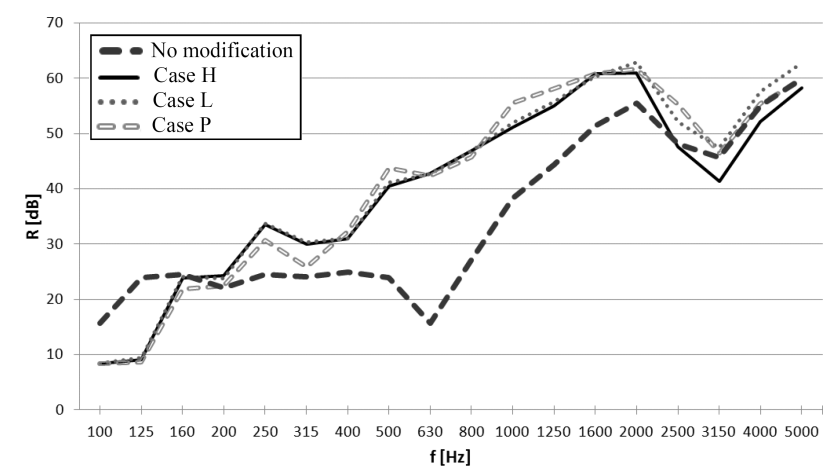

Fig. 17. Sound insulation curve for modified CSIP from FEM: influence of glass wool layer position from Table 3 ( $R$ - sound reduction index, $f$ - frequency).

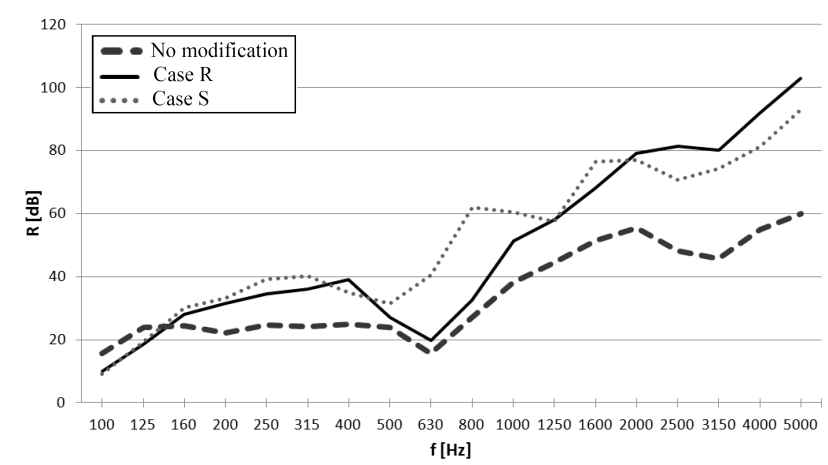

Fig. 18. Sound insulation curve for modified CSIP from FEM: double panel CSIP from Table $3(R$ - sound reduction index, $f$ - frequency). 
The suggested modifications of the EPS core are able to improve the sound insulation of composite panels, in particular with the aid of a glass wool layer. For the modified CSIP with the air void inserts $20 \mathrm{~cm}$ wide (case 'A'), the first resonant frequency was shifted from $630 \mathrm{~Hz}$ down to $500 \mathrm{~Hz}$ and the sound reduction index $R$ increased from $15.7 \mathrm{~dB}$ up to $29.2 \mathrm{~dB}$ in the area of the mass-spring-mass resonance (Fig. 11). However, this modification decreased the sound insulation at the low frequency range $(250-315 \mathrm{~Hz})$ and decreased the sound reduction index $R_{A 1}$ from $26.1 \mathrm{~dB}$ (original panel) down to $27.3 \mathrm{~dB}$ (Table 4). In turn, the index $R_{A 2}$ was the same (Table 4 ). On the other hand, the $40 \mathrm{~cm}$ wide air void inserts (case ' $\mathrm{C}$ ') allowed for a sound insulation growth in a broad frequency range (from $160 \mathrm{~Hz}$ up to $1000 \mathrm{~Hz}$ ). The index $R$ increased from $15.7 \mathrm{~dB}$ up to $23.6 \mathrm{~dB}$ in the area of a symmetric coincidence frequency. However, the low frequency acoustic insulation was smaller. An increase of the both indexes $\left(R_{A 1}, R_{A 2}\right)$ by $3.5-1 \mathrm{~dB}$ was observed (Table 4).

When air void inserts were replaced by a glass wool (cases 'B' and 'D'), the sound insulation increased obviously with the growing width of wool inserts. An increase of the sound insulation at the broad frequency range was obtained in the cases ' $\mathrm{B}$ ' and ' $\mathrm{D}$ ' (Fig. 12) due to the weight augmentation. Thus, a noticeable increase by $2.5-6.1 \mathrm{~dB}$ was obtained for the ratings $R_{A i}$.

A significant positive effect of an additional damping layer (glass wool) on the shift of the resonant frequency is showed in Fig. 13. All modified panels (cases 'E', 'F', 'G' and 'H') had always a greater sound insulation in a mid-region of the frequency band. The addition of the $1 \mathrm{~cm}$ wide damping layer (case ' $\mathrm{E}$ ') shifted the low resonant frequency to $315 \mathrm{~Hz}$ and increased $R$ up to $43.8 \mathrm{~dB}$ in the area of resonant frequency $(f=630 \mathrm{~Hz})$. Despite of a sound insulation increase in the range of mid-frequencies, the sound reduction indexes $R_{A 1}$ and $R_{A 2}$ were greater by $4.5 \mathrm{~dB}$ and $2.5 \mathrm{~dB}$ only (Table 4). This is related to the smaller sound insulation in a low frequency range. The panel with the additional $1 \mathrm{~cm}$ thick damping layer (case 'E') was the most advantageous because it was characterized by the greatest growth of sound insulation rating $R_{A 2}$. The differences in the sound insulation ratings $R_{A i}$ between the panel with the $8 \mathrm{~cm}$ (case ' $\mathrm{H}$ ') and the basic configuration were $6.0 \mathrm{~dB}$ and $0.9 \mathrm{~dB}$, respectively. Thus, the greatest increase of the sound rating $R_{A 1}$ was occurred in the case ' $\mathrm{H}$ ' (8-cm-thick additional glass wool layer). On the other hand, the greatest growth of the sound rating $R_{A 2}$ happened in the case ' $\mathrm{E}$ ' (1-cm-thick additional glass wool layer).

The influence of an additional layer location on sound insulation curves is showed in Figs. 14-17. The overall thickness of additional glass wool layers was the same. Three layer types were taken into account: single mid-layer, single lateral layer and double lateral layer (Fig. 10). The effect of the location was negligible for the thickest glass wool layer (Fig. 17). The application of a double layer (cases ' $\mathrm{M}$ ', ' $\mathrm{N}$ ', 'O' and 'P' in Fig. 10) is recommended in order to increase the sound insulation in a medium and high frequency range.

A strong improvement of the acoustic behaviour was also obtained with double CSIPs (cases 'R' and 'S') (Fig. 18) that was mainly related with the weight increase and the presence of a continuous glass wool layer between panels (Fig. 10). A non-symmetric case 'R' had the greatest sound insulation indexes (Table 4). It was more advantageous than the symmetric case ' $\mathrm{S}$ ' due to different resonance frequencies of single layers in the case ' $\mathrm{R}$ '.

Further studies will be carried out on modifications of CSIPs to improve their acoustic properties by taking into account technical and economical aspects of a manufacturing process.

\section{Conclusions}

The following conclusions can be derived based on our experimental and numerical results concerning the airborne sound insulation of CSIPs:

- The airborne sound insulation of panels is low and does not satisfy the standard requirements. It is caused by the presence of a light compressible EPS core that contributes to resonant frequencies at $630 \mathrm{~Hz}$ and $3150 \mathrm{~Hz}$, indicated by local dips in the sound insulation curve.

- A very good agreement with experimental sound reduction ratings was obtained using a standard steady-state acoustic 2D approach in ABAQUS. When using a dynamic transient 2D FE model, a satisfactory agreement with the experimental curve was also achieved. Thus, this numerical model turned out to be an efficient tool to predict the sound insulation of building partitions and to develop products with improved acoustic properties. The $2 \mathrm{D}$ results were confirmed by the $3 \mathrm{D}$ computation results for frequencies less than $<1250 \mathrm{~Hz}$ (steady-state and transient simulations).

- The analytical formula provides a good approximation of the sound reduction curve in a low and medium frequency range $(f<1600 \mathrm{~Hz})$.

- The acoustic properties can be improved by the presence of air void inserts, glass wool inserts, glass wool layers or by connecting two panels through a wool layer that may lift the sound insulation curve and shift the resonant frequencies. The most effective improvement in terms of the $R_{A 1}$ rating was the wool layer with the thickness of $1 \mathrm{~cm}$. In turn, the most effective improvement in terms of the $R_{A 2}$ rating was the wool layer with the thickness of $8 \mathrm{~cm}$. 


\section{Acknowledgement}

The FE simulations were performed on computers of the Academic Computer Centre in Gdańsk TASK.

Research work has been carried out within the project: "Performance of development works in order to introduce into market an innovative system composed of light structural panels to residential buildings' structure and acoustic baffles" financed partly by the European Union (POIG.01.04.00-24-073/09-00) and the project "Innovative complex system solution for energy-saving residential buildings of a high comfort class in an unique prefabricated technology and assembly of composite panels" financed by National Centre of Research and Development NCBR (No. R1/INNOTECH-K1/IN1/59/155026/NCBR/12).

\section{References}

1. ABAQUS (2010a), Theory Manual, Ver. 6.10.

2. ABAQUS (2010b), Analysis User's Manual, Ver. 6.10.

3. Amichi K., Atalla N., Ruokolainen R. (2010), A new $3 D$ finite element sandwich plate for predicting the vibroacoustic response of laminated steel panels, Finite Elements in Analysis and Design, 46, 1131-1145.

4. Del Coz Díaz J.J., Rabanal P.J.A., Nieto P.J.G., LóPEz M.A.S. (2010a), Sound transmission loss analysis through a multilayer lightweight concrete hollow brick wall by FEM and experimental validation, Building and Environment, 45, 2373-2386.

5. Del Coz Díaz J.J., Nieto F.P.G., Rabanal F.P., SiERRA J.L.S. (2010b), Optimization of an acoustic test chamber involving the fluid-structure interaction by FEM and experimental validation, Meccanica, 45, 704-722.

6. Cremer L., Heckl M. (1988), Structure-borne sound, Springer Verlag, Berlin.

7. HöKmaRK B. (2007), Acoustic analysis of loudspeakers cavity including viscothermal effects, Master's Dissertation, Lund University.

8. ISO 140-1 (1997), Acoustics - Measurement of sound insulation in buildings and of building elements Part 1: Requirements for laboratory test facilities with suppressed flanking transmission.

9. ISO 140-3 (1995), Acoustic - Measurement of sound insulation in buildings and of building elements - Part 3: Laboratory measurements of airborne sound insulation of building elements.

10. ISO 140-4 (1998). Acoustics - Measurement of sound insulation in buildings and of building elements Part 4: Field measurements of airborne sound insulation between rooms.

11. ISO 717-1 (1996), Acoustics - Rating of sound insulation in buildings and of building elements - Part 1: Airborne sound insulation.
12. Krakers L.A. (2009), Parametric fuselage design Integration of mechanics and acoustic $\& 5$ thermal insulation, PhD Thesis, Delft University.

13. LANG M.A., Dym C.L. (1975), Optimal acoustic design of sandwich panels, Journal of Acoustical Society of America, 57, 1481-1487.

14. Makris S.E., Dym C.L., MacGregor Smith J. (1986), Transmission loss optimization in acoustic sandwich panels, Journal of Acoustical Society of America, 79, 1833-1843.

15. Maluski S., GibBs B.M. (2004), The effect of construction material, contents and room geometry on the sound field in dwellings at low frequencies, Applied Acoustics, 65, 31-44.

16. Naify Ch. J., Huang Ch., Sneddon M., Nutt S. (2011), Transmission loss of honeycomb sandwich structures with attached gas layers, Applied Acoustics, 72, 71-77.

17. NAOKI K., TAKayasu U. (2007), Improvements to the Johnson-Allard model for rigid-framed fibrous materials, Applied Acoustics, 68, 1468-1484.

18. Okuzono T., Otsuru T., Tomiku R., Okamoto N. (2010), Fundamental accuracy of time domain finite element method for sound-field analysis of rooms, Applied Acoustics, 71, 940-946.

19. Papadopoulos C.I. (2002), Development of an optimised, standard compliant procedure to calculate sound transmission loss: design of transmission rooms, Applied Acoustics, 63, 1003-1029.

20. Papadopoulos C.I. (2003), Development of an optimized, standard compliant procedure to calculate sound transmission loss: numerical measurements, Applied Acoustics, 64, 1069-1085.

21. Peters S.T. (1998), Handbook of composites, Chapman \& Hall, London.

22. PN-B-02151-3 (1999), Building Acoustics - Noise protection in buildings - Sound insulation of buildings and building elements - Requirements [in Polish].

23. Shengchun W., Zhaoxiang D., Weidong D.S. (2010), Sound transmission loss characteristics of unbounded orthotropic sandwich panels in bending vibration considering transverse shear deformation, Composite Structures, 92, 12, 2885-2889.

24. Smakosz L., Wawrzynowicz A., KrzaczeK M., Tejchman J. (2012), Experimental and numerical evaluation of composite structural insulated wall panels, Proc. 15th European Conference on Composite Materials ECCM 15, Venice, Italy.

25. Smakosz L., Tejchman J. (2014), Evaluation of strength, deformability and failure mode of composite structural insulated panels, Materials and Design, 54C, 1068-1082. 
26. Szudrowicz B., Żuchowicz-Wodnikowska I., TOMCZYK P. (2002), Sound insulation properties of building barriers and their components [in Polish], Building Research Institute (ITB), Warszawa.

27. TARnow V. (2005), Dynamic measurements of the elastic constants of glass wool, J. Acoust. Soc. Am., 118, 6, 3672-3678.

28. WANG T., Li S., NutT S.R. (2009), Optimal design of acoustical sandwich panels with a genetic algorithm, Applied Acoustics, 70, 3, 416-425.
29. Wen-Chao H., Chung-Fai N. (1998), Sound insulation improvement using honeycomb sandwich panels, Applied Acoustics, 53, 1-3, 163-177.

30. Vigran T.E. (2008), Building Acoustics, Taylor \& Francis, London and New York.

31. XenAKI V.K. (2005), Experimental investigation of the mechanical behaviour of EPS geofoam under static and dynamic/cyclic loading, PhD Thesis, University of Patras. 